JGG 2022:70:88-98

doi: $10.36150 / 2499-6564-N 440$

\title{
Disentangling the impact of COVID-19 infection on clinical outcomes and preventive strategies in older persons: an Italian perspective
}

Received: September 21, 2021

Accepted: November 1, 2021

\section{Correspondence}

Angela Marie Abbatecola

Azienda Sanitaria Locale (ASL) di Frosinone, Alzheimer's Disease Day Center, via Ponte Melfa Atina, 03042 Frosinone, Italy.

Email: angela_abbatecola@yahoo.com, angelamarie.abbatecola@aslfrosinone.it

How to cite this article: Abbatecola AM, Antonelli Incalzi R, Malara A, et al. Disentangling the impact of COVID-19 infection on clinical outcomes and preventive strategies in older persons: an Italian perspective. Journal of Gerontology and Geriatrics 2022;70:88-98. https://doi. org/10.36150/2499-6564-N440

(C) Copyright by Società Italiana

di Gerontologia e Geriatria (SIGG)

\section{(c) (1) () $\odot$}

This is an open access article distributed in accordance with the CC-BY-NC-ND (Creative Commons Attribution-NonCommercial-NoDerivatives 4.0 International) license. The article can be used by giving appropriate credit and mentioning the license, but only for non-commercial purposes and only in the original version. For further information: https://creativecommons.org/licenses/by-nc-nd/4.0/deed.en
Angela Marie Abbatecola ${ }^{1,2}$, Raffaele Antonelli Incalzi ${ }^{2,3}$, Alba Malara ${ }^{2,4}$, Annapina Palmieri ${ }^{5}$, Anna Di Lonardo ${ }^{5}$, Gilda Borselli ${ }^{2}$, Marcello Russo', Marianna Noale ${ }^{6}$, Stefano Fumagalli ${ }^{7}$, Pietro Gareri ${ }^{8}$, Enrico Mossello ${ }^{7}$, Caterina Trevisan ${ }^{9,10}$, Stefano Volpato ${ }^{2,10}$, Fabio Monzani ${ }^{2,11}$, Alessandra Coin ${ }^{9}$, Giuseppe Bellelli2,12, Chukwuma Okoye ${ }^{11}$, Susanna Del Signore ${ }^{13}$, Gianluca Zia ${ }^{13}$, Elisa Bottoni ${ }^{14}$, Carmine Cafariello ${ }^{15}$, Graziano Onder ${ }^{5}$, GeroCovid Observational ${ }^{16}$ \& GeroCovid Vax Group ${ }^{17}$

${ }^{1}$ Azienda Sanitaria Locale (ASL) Alzheimer's Disease Day Clinic, Frosinone, Italy; ${ }^{2}$ Italian Society of Gerontology and Geriatrics (SIGG), Florence, Italy; ${ }^{3}$ Unit of Geriatrics, Department of Medicine, Campus Bio-Medico University and Teaching Hospital, Rome, Italy; ${ }^{4}$ ANASTE-Humanitas Foundation, Rome, Italy; ${ }^{5}$ Department of Cardiovascular, Endocrine-Metabolic Diseases and Aging, Istituto Superiore di Sanità, Rome, Italy; ${ }^{6}$ Aging Branch, Neuroscience Institute, National Research Council, Padua, Italy; ${ }^{7}$ Geriatric Intensive Care Unit, Department of Experimental and Clinical Medicine, University of Florence, Italy; ${ }^{8}$ Center for Cognitive Disorders and Dementia - Catanzaro Lido, ASP Catanzaro, Italy; ${ }^{9}$ Geriatrics Division, Department of Medicine (DIMED), University of Padua, Italy; ${ }^{10}$ Department of Medical Science, University of Ferrara, Italy; ${ }^{11}$ Geriatrics Unit, Department of Clinical and Experimental Medicine, University of Pisa, Pisa, Italy; ${ }^{12}$ School of Medicine and Surgery, University of Milano-Bicocca and Acute Geriatric Unit, San Gerardo Hospital, Monza, Italy; ${ }^{13}$ Bluecompanion Ltd, London, UK; ${ }^{14}$ Centro Benessere (Rehabilitation center) Frosinone, Italy; ${ }^{15}$ Geriatrics Outpatient Clinic and Territorial Residences, Italian Hospital Group, Rome, Italy; ${ }^{16,17}$ Group members are listed in the appendix

Italy was one of the first western countries to embrace the first wave of COVID-19 and undergo detrimental outcomes in older adults in different clinical settings, especially in those with comorbidity and frailty. In addition, older nursing home $(\mathrm{NH})$ residents had significantly higher mortality rates most likely due to the increased susceptibility of infection due to combined physical vulnerability and risks linked to the $\mathrm{NH}$ living environment itself.

Different reports throughout Italy have rapidly highlighted selected outcomes related to COVID-19 in older patients being treated in acute and long-term care (LTC) settings. However, the majority of these studies are single center studies. Thus, it remains fundamental to collect large data from prospective based-population studies in order to identify preventive and therapeutic COVID-19 risk/protective factors correlated with COVID-19 health status outcomes.

In this commentary paper, we will discuss different Italian reports according to clinical settings and highlight the importance of GeroCovid Observational and GeroCovid Vax, two large population based prospective studies in Italy.

Key words: COVID-19, Italy, vaccines in long term care, elderly, depression 


\section{INTRODUCTION}

The Coronavirus disease 2019 (COVID-19) pandemic caused by severe acute respiratory syndrome coronavirus (SARS-CoV-2) has caused high rates of morbidity and mortality on a worldwide scale. Italy was one of the first countries to embrace the impact of the first wave of COVID-19 in older adults with negative outcomes, especially in frail elders. During the first wave of the pandemic, older Italians especially with frailty ${ }^{1}$, showed to be the extremely vulnerable to mortality with overall fatality rates reaching $26 \%$ in acute care ${ }^{2}$ and $33 \%$ in long-term care settings ${ }^{3}$.

During the month of March 2020, case fatality rates were $20.2 \%$ in those over 80 years with a greater vulnerability in men ${ }^{4}$. Similar patterns quickly followed across different countries worldwide. For example, one US report found that in 5700 COVID-19 cases, case fatality rates were over $40 \%$ in those over the age of 80 years during hospitalization, raising concerns on very old vulnerable people ${ }^{5}$. Even though it remains unclear the role played by common comorbidities, older age continues to be the highest lethality risk factor since the beginning of the pandemic.

The extremely high mortality rates linked to COVID-19 in those living in Nursing Homes $(\mathrm{NH})$ caused a severe worldwide crisis in $\mathrm{NH}$ organization and functioning. The combinations of vulnerable older persons (with frailty syndrome and comorbidities) along with $\mathrm{NH}$ living environment (group gatherings, more than one person per room, limited personal protection equipment, etc.) underline the so-called "Perfect Storm" for COVID-19 transmission ${ }^{6}$. In Italy, fatality rates reached 33\% in long-term care settings ${ }^{3}$. Worldwide data in approximately 50 studies found that long-term care residents had an attack rate of 45\% for COVID-19 and a death rate of $23 \%{ }^{7}$. Indeed, the urgent need for answers related to COVID-19 prognosis in aging elders has prompted researchers to aim at identifying related risk factors and innovative therapeutic measures.

One Italian report underlined that specific combinations of comorbidities during COVID-19 infection were associated with in-hospital mortality in older persons (mean age 79 years) ${ }^{8}$. These authors underlined that the following combinations: i) ischemic heart disease and atrial fibrillation; ii) atrial fibrillation and heart failure; iii) atrial fibrillation and stroke; iv) heart failure and COPD; v) stroke and dementia, and vi) type 2 diabetes and obesity occurred more frequently than expected in those dying with COVID-19, thus, indicating a potential role of these clinical profiles in the chain of events that led to death in those who contracted SARS-CoV-2. At the moment, large prospective longitudinal studies in older persons can successfully provide important indicators needed to better understand this unraveling coronavirus.

In this commentary, we will discuss diverse Italian findings related to COVID in older persons. We will highlight the importance of prospective population based studies that are currently providing evidence for potential risk factors associated with COVID-19. The first study is the GeroCovid Observational Study, a multicenter and multi-setting study that is currently disentangling questions in both acute and long-term care (LTC), as well as in outpatient care. The second study is the GeroCovid Vax study dedicated to identifying the safety and efficacy of COVID-19 vaccinations in older persons living in NHs. The actual inclusion of older persons in the initial clinical trials for evaluating vaccine safety and efficacy were not representative enough of the geriatric population ${ }^{\text {9; }}$ thus, understanding their role in older vulnerable individuals will provide important knowledge to the available literature.

\section{METHODS}

\section{Study POPULATION}

GeroCovid Observational included persons $\geq 60$ years of age with or at risk of COVID-19 willing to participate in the study. Data were collected retrospectively and prospectively from March 1, 2020 to December 31, 2020. The study has been described elsewhere ${ }^{10}$. The Italian Society of Gerontology and Geriatrics (SIGG), in collaboration with the Norwegian Geriatrics Society founded the GeroCovid Observational Study, a multicenter and multi-setting study that is currently providing evidence for risk factors in both acute and long-term care (LTC), as well as in outpatient care settings. GeroCovid Observational is also investigating clinical presentation of infection and clinical outcomes, including changes in health status, hospitalizations, and death. GeroCovid Vax Study began in January 2021. The Italian National Institute of Health (Istituto Superiore di Sanità, ISS; Rome, Italy) and the Italian Society of Gerontology and Geriatric Medicine (SIGG; Florence, Italy) formed the GeroCovid Vax study group to investigate the safety and efficacy of COVID-19 vaccination in older adults living in $\mathrm{NHs}$ throughout Italy. In a subpopulation, both humoral and immune cellular responses are being tested to identify an adequate level of vaccine protection. Enrollment period was from January to June 30th, 2021. There are planned follow-up periods to test for changes in clinical and biochemical data. Timed follow up visits are planned at specific intervals for a 12-month follow up observational period. Information regarding any adverse events from COVID-19 vaccinations are 
reported, as well as any changes in cognitive, functional and routine blood parameters are registered (Tab. I).

The data collection for both studies use the same platform provided by BlueCompanion (United Kingdom, France) ${ }^{11}$. In particular, the GeroCovid e-Registry was developed by adapting a proprietary electronic platform (e-Trajectories). In March 2020, in conjunction with the COVID-19 pandemic, BlueCompanion provided their health data collection system for the GeroCovid initiative. E-Trajectories and GeroCovid adaptation are based on the CleanWeb engine produced by Telemedicine Technologies (Boulogne-Billancourt, France), embedded in a dedicated web platform designed for integrating data from different sources. All data are recorded on web servers located in the EU (France and Poland). ICT operations are compliant with the European General Data Protection Regulation (GDPR) and with the relevant international standards for clinical trials (ISO 9001 certification and FDA CFR 21 part 11). Bluecompanion continuously provides training sessions for investigators from each investigational site, as well as technical support. Data collection is under constant quality control and investigators are required, when necessary to verify data entry. All individual clinical data were anonymized before data entry.

\section{ETHICAL ASPECTS}

The Campus Bio-Medico University Ethical Committee approved the overarching protocol of the GeroCovid Observational study in April 2020. All participating investigational sites gained approval from their local Ethical Committee review board. Informed consent was necessary to participate in the study.

\section{RESULTS AND ONGOING INVESTIGATIONS}

\section{GeroCovid Observational Study}

3396 participants were included per protocol by 86 Italian investigational sites, (plus 148 from 3 sites in Norway). Recent reports from different settings have

Table I. General characteristics of GeroCovid Observational and GeroCovid Vax Studies.

\begin{tabular}{|c|c|c|}
\hline & GeroCovid Observational & GeroCovid Vax \\
\hline \multirow[t]{2}{*}{ Age } & $\geq 60$ years of age & $\geq 60$ years of age \\
\hline & - with COVID-19 - no COVID-19 & - no COVID-19 \\
\hline \multirow[t]{7}{*}{ Study Aims } & - assess changes in COVID risk profile & - assess the safety of COVID-19 vaccines \\
\hline & $\begin{array}{c}\text { - assess the impact on physical, cognitive, } \\
\text { psychological \& behavioral status in non COVID-19 } \\
\text { participants }\end{array}$ & $\begin{array}{l}\text { - assess adverse side effects following vaccine } \\
\text { inoculation }\end{array}$ \\
\hline & \multirow[t]{3}{*}{$\begin{array}{l}\text { - assess clinical \& functional outcomes following } \\
\text { COVID-19 hospitalization }\end{array}$} & $\begin{array}{l}\text { - assess clinical \& functional changes over time } \\
\text { following vaccination }\end{array}$ \\
\hline & & - assess COVID-19 following vaccination \\
\hline & & - assess efficacy (monitor immune response) \\
\hline & $\begin{array}{c}\text { - identify significant measures in homecare \& } \\
\text { outpatient services }\end{array}$ & \\
\hline & $\begin{array}{l}\text { - identify measures in LTC to prevent \& protect against } \\
\text { COVID-19 }\end{array}$ & \\
\hline Settings & $\begin{array}{l}\text { Acute wards, outpatient clinics (memory, post } \\
\text { hospitalization from COVID-19), Homecare assistance, } \\
\text { LTC (assisted living, retirement homes, NHs) }\end{array}$ & LTC (NH, Retirement home) \\
\hline $\begin{array}{l}\text { Functional, cognitive, } \\
\text { comorbidity } \\
\text { parameters }\end{array}$ & ADL, IADL, CIRS, MMSE, GDS, physical activity & ADL, IADL, CIRS, MMSE, GDS, physical activity \\
\hline $\begin{array}{l}\text { Frailty anamnestic } \\
\text { parameters }\end{array}$ & Frailty criteria ${ }^{20-21}$ & Frailty criteria ${ }^{20-21}$ \\
\hline $\begin{array}{l}\text { Biochemical } \\
\text { parameters }\end{array}$ & Blood/urine analyses & Blood samples \\
\hline Drug use monitoring & ATC classification & ATC classification \\
\hline Outcome & $\begin{array}{l}\text { Type of outcome }{ }^{10} \text { (infection, death, hospitalization, } \\
\text { changes in clinical, cognitive \& functional parameters) }\end{array}$ & $\begin{array}{l}\text { Adverse events (infection, death, hospitalization, } \\
\text { other) immune response }\end{array}$ \\
\hline Data entry method & E-registry (BlueCompanion, France) ${ }^{11}$ & E-registry (BlueCompanion, France) ${ }^{11}$ \\
\hline
\end{tabular}


been published using data from the electronic registry. One study from the acute care setting, showed approximately $22 \%$ of COVID-19 patients had an Atrial Fibrillation (AF) and in-hospital mortality was significantly higher in the AF group (36.9 vs $27.5 \%, p=0.015)^{12}$. The authors also found that i) the use of preadmission and in-hospital oral anticoagulation in older patients with $\mathrm{AF}$ was correlated with improved survival; ii) inhospital low-molecular-weight heparin therapy, when following oral anticoagulation at home, was associated with reduced mortality in older patients with $\mathrm{AF}$ and COVID-19; iii) a better preadmission functional profile is related to higher survival in older adults with $\mathrm{AF}$ and COVID-19.

Another report sampled 90 patients in outpatient and homecare services setting and tested for the role of preserving health status through telephone and video calls during lockdown ${ }^{13}$. They concluded that Activities of Daily Living (ADL), cognitive functioning using the Mini Mental State Examination (MMSE) and quality of life using the European Quality of Life scale (EuroQoL) scores (ADL: $2.27 \pm 1.65$ vs $1.98 \pm 1.72, p=0.001$; MMSE: $15.59 \pm 7.83$ vs $14.49 \pm 7.96, p<0.001$; EuroQol: $42.22 \pm 11.88$ vs $40.0 \pm 12.45, p=0.005)$ significantly worsened over time from TO (March 2020) and T1 (May 2020), while no significant changes were found in Instrumental activities of daily living (IADL), Geriatric Depression Scale (GDS) and Cumulative Illness Rating Scale (CIRS) scores ${ }^{13}$. Even though the majority of patients were clinically stable over time, behavioral changes were found in $24.4 \%$ of patients and anxiety and insomnia in $17.7 \%$ of patients ${ }^{13}$.

A new preliminary report (in press), carried-out on 168 patients afferent to 10 different Italian Centers for cognitive decline and dementia (CDCD), investigated the impact of lockdown on psychological well-being of individuals with cognitive deterioration. Symptoms of anxiety (28.8\%), depression (48\%), and psychological stress (24.8\%) were observed in these patients who were found to experience psycho-affective disorders according to their pre-quarantine cognitive functioning. In particular, during the first lockdown, individuals cognitively more impaired showed more severe depressive and anxious symptoms, compared to those with better cognitive functioning ${ }^{14}$.

Four additional studies currently are under evaluation, related to the age-related changes in clinical presentation, the chest tomography pattern of COVID-19 in the very old, impact of the lockdown on the health status of demented patients and the clinical and epidemiological features of COVID-19 in NHs. All investigations testify the focus of GeroCovid Observational on the frail population directly or indirectly suffering form COVID-19.

\section{GeroCovid Vax study}

The Ethical Committee of the Spallanzani Hospital approved the GeroCovid Vax study protocol. Informed consent was also necessary to participate. The study began in January 2021 and ended enrollment as of June 30, 2021. Currently, per protocol inclusion data are available in 3014 participants from 76 Nursing homes all over Italy. Of these, 685 participants have undergone blood testing for humoral and cellular immunity testing which are necessary to understand the efficacy of the immune response in old age. As of August 12, 2021 the clinical follow-up is still ongoing, however enrollment has ceased. Clinical data collections are being performed ( 7 days prior to the first vaccine dose, 7 days following the second dose, 2-, 6-, and 12-months following the first dose). The study will be concluded in the second quarter of 2022.

\section{DISCUSSION}

The COVID-19 pandemic has stimulated numerous research protocols in geriatric medicine in order to identify and to improve health outcomes from COVID-19 infection, as well as to understand the benefits related to vaccine use. Older and in particular, frail older adults have continuously demonstrated to be at a significantly higher risk for negative health outcomes. A meta-analysis regarding mortality in older Italians with a COVID-19 infection found that dementia, diabetes, chronic kidney disease and hypertension were the main comorbidities associated with death in study sizes ranging from 18 to 1591 individuals ${ }^{15}$. These authors underlined that at the time of admission, clinical frail severity, as well as the presence of $>3$ comorbidities, were significantly associated with an increased overall death rate. In comparison to the GeroCovid Observational data regarding in-hospital mortality, a newly published report based in Italy confirmed the importance of clinical functional characteristics at admission on outcomes in older persons ${ }^{16}$. Both GeroCovid Observational and GeroCovid Vax specifically include physical and cognitive functioning parameters, as well as frailty markers.

At the moment, GeroCovid Observational study holds a particular role on identifying risk factors related to outcomes from COVID-19 due to study design. GeroCovid Observational is continuously monitoring and updating the health status of study participants prospectively in order to gain knowledge regarding outcomes in diverse clinical settings, while GeroCovid Vax is providing an important basis for the use and safety of COVID-19 vaccinations in vulnerable elders living in $\mathrm{NHs}$.

Lockdown measures held an overall negative impact on socialization in older persons. As previously mentioned 
findings from GeroCovid Observational show a significant increase in anxiety and insomnia using telemedicine techniques to check and follow-up patient health status ${ }^{13}$. Interestingly, another Italian study testing the impact of social distancing during the first wave quarantine/self-isolation (31 \pm 5 mean days) in 334 participants, using an online survey, found that a higher anger score was significantly and negatively related to age, and scores on the Brief Resilience Score and Coping Scale, whereas a significant and positive relationship was found with the fear of getting infected and female gender ${ }^{17}$. The negative impact on social status related to COVID-19 is raising serious concern for long-term effects on health status. GeroCovid Observational may provide the necessary answers.

Additional strengths of GeroCovid Observational include the involvement of multiple clinical settings with the eregistry platform that ensures for a high-quality data entry from each setting. In older persons, GeroCovid Observational has provided and will continue to provide data regarding: i) disease infection onset symptoms; ii) factors related to clinical course of COVID-19; iii) effects on health status of older persons at risk of COVID-19. Since the implementation of anti-COVID-19 vaccines in December 2020, aggregated public data have shown decreases in the incidence of cases of SARS-CoV-2 infection and related deaths ${ }^{18}$. However, lower incident cases might also reflect improved $\mathrm{NH}$ organization reducing virus transmission spread. Indeed, by understanding vaccine effectiveness in $\mathrm{NH}$ residents, who were not included in clinical trials and who often have reduced immune responses, the GeroCovid Vax study will disentangle numerous doubts of vaccine efficacy and safety ${ }^{19}$.

In conclusion, GeroCovid and GeroCovid Vax represent an example of real life research applied to the most vulnerable fraction of the population exposed to COVID-19. Interestingly, they rely upon a sophisticated and high quality methodological approach to achieve in a standardized way the relevant information from different clinical settings. By standardizing the assessment instruments, they make possible to identify setting specific features of the pandemic. Eventually, the integrated clinical and laboratory approach to the immunization policy will gain insight into the immunologic response of the most frail and multimorbid people.

\section{Ethical consideration}

The Campus Bio-Medico University Ethical Committee approved the overarching protocol of the GeroCovid Observational study in April 2020. All participating investigational sites gained approval from their local Ethical Committee review board. Informed consent was necessary to participate in the study.
The Ethical Committee of the Spallanzani Hospital approved the GeroCovid Vax study protocol. Informed consent was necessary to participate.

\section{Acknowledgement}

The Authors would like to thank the members of the Bluecompanion team: Alessandro Loria, Simonetta Demarie, Stefania Del Signore e Patrizia Angelucci.

\section{Funding}

The GeroCovid Observational study did not receive any funding. The GeroCovid Vax study was funded by a grant from the Italian Medicines Agency (AIFA).

\section{Conflict of interest}

The Authors declare no conflict of interest.

\section{References}

1 Abbatecola AM, Antonelli-Incalzi R. Editorial: COVID-19 spiraling of frailty in older Italian patients. J Nutr Health Aging 2020;24:453-455. https://doi.org/10.1007/ s12603-020-1357-9

2 Ruscica M, Macchi C, lodice S, et al. Prognostic parameters of in-hospital mortality in COVID-19 patients - an Italian experience. Eur J Clin Invest 2021;51:e13629. https:// doi.org/10.1111/eci.13629

3 Lombardo FL, Bacigalupo I, Salvi E, et al.; Italian National Institute of Health Nursing Home Study Group. The Italian national survey on Coronavirus disease 2019 epidemic spread in nursing homes. Int J Geriatr Psychiatry 2021;36:873-882. https://doi.org/10.1002/gps.5487

4 Onder G, Rezza G, Brusaferro S. Case-fatality rate and characteristics of patients dying in relation to COVID-19 in Italy. JAMA 2020;323:1775-1776. https://doi.org/doi: 10.1001/jama.2020.4683. Erratum in: JAMA 2020;Apr 28;323(16):1619.

5 Richardson S, Hirsch JS, Narasimhan M, et al.; the Northwell COVID-19 Research Consortium. Presenting characteristics, comorbidities, and outcomes among 5700 patients hospitalized with COVID-19 in the New York City Area. JAMA 2020;323:2052-2059. https://doi. org/10.1001/jama.2020.6775 Erratum in: JAMA 2020;May 26;323(20):2098.

6 Ouslander JG, Grabowski DC. COVID-19 in nursing homes: calming the perfect storm. J Am Geriatr Soc 2020;68:2153-2162. https://doi.org/10.1111/jgs.16784

7 Hashan MR, Smoll N, King C, et al. Epidemiology and clinical features of COVID-19 outbreaks in aged care facilities: a systematic review and meta-analysis. EClinicalMedicine. 2021;33:100771. https://doi.org/10.1016/j. eclinm.2021.100771

8 Vetrano DL, Tazzeo C, Palmieri L, et al.; Italian National Institute of Health Covid-Mortality Group. Comorbidity status of deceased COVID-19 in-patients in Italy. Aging Clin Exp Res 2021;33:2361-2365. https://doi.org/10.1007/ s40520-021-01914-y 
9 Antonelli Incalzi R, Trevisan C, Del Signore S, et al. H. Are vaccines against COVID-19 tailored to the most vulnerable people? Vaccine 2021;39:2325-2327. https://doi. org/10.1016/j.vaccine.2021.03.066

10 Trevisan C, Del Signore S, Fumagalli S, et al.; GeroCovid Working Group. Assessing the impact of COVID-19 on the health of geriatric patients: the European GeroCovid Observational Study. Eur J Intern Med 2021;87:29-35. https://doi.org/10.1016/j.ejim.2021.01.017

11 https://www.bluecompanion.eu (accessed July 1, 2021).

12 Fumagalli S, Trevisan C, Del Signore S, et al.; GeroCovid Working Group. COVID-19 and atrial fibrillation in older patients: does oral anticoagulant therapy provide a survival benefit? An insight from the GeroCovid Registry. Thromb Haemost 2021;May 7. https://doi. org/10.1055/a-1503-3875 [Epub Ahead of Print]

13 Gareri P, Fumagalli S, Malara A, et al.; GeroCovid Ambulatory Study Group. Management of older outpatients during the COVID-19 pandemic: the GeroCovid Ambulatory Study. Gerontology 2021;Jun 28:1-6. https://doi. org/10.1159/000516969 [Epub Ahead of Print]

14 Coin A, Devita M, Trevisan C, et al. Psychological wellbeing of older adults with cognitive deterioration during quarantine: preliminary results from the GeroCovid Initiative. Front Med 2021: 8:715294. https://doi.org/10.3389/ fmed.2021.715294 (in press)

15 Alves VP, Casemiro FG, Araujo BG, et al. Factors associated with mortality among elderly people in the COVID-19 pandemic (SARS-CoV-2): a systematic review and metaanalysis. Int J Environ Res Public Health 2021;18:8008. https://doi.org/10.3390/ijerph18158008
16 Covino M, Russo A, Salini S, et al. Frailty assessment in the emergency department for risk stratification of COVID-19 patients aged $\geq 80$ years. J Am Med Dir Assoc 2021:S1525-8610(21)00643-5. https://doi.org/10.1016/j. jamda.2021.07.005

17 Maggi G, Baldassarre I, Barbaro A, et al. Mental health status of Italian elderly subjects during and after quarantine for the COVID-19 pandemic: a cross-sectional and longitudinal study. Psychogeriatrics 2021;21:540-551. https://doi. org/10.1111/psyg.12703

18 Chidambaram P, Garfield R, Neuman T, et al. New COVID-19 cases and deaths among nursing home residents have dropped since vaccinations began. Kaiser Family Foundation. March 3, 2021 (https://www.kff.org/coronavirus-covid-19/slide/new-covid-19-cases-and-deathsamong-nursing-home-residents-have-dropped-sincevaccinations-began/).

19 White EM, Yang X, Blackman C, et al. Incident SARS-CoV-2 infection among mRNA-vaccinated and unvaccinated nursing home residents. N Engl J Med 2021;385:474-476. https://doi.org/10.1056/NEJMc2104849

20 Pedone C, Costanzo L, Cesari M, et al. Are performance measures necessary to predict loss of independence in elderly people? J Gerontol A Biol Sci Med Sci 2016;71:8489. https://doi.org/10.1093/gerona/glv096

21 Fried LP, Tangen CM, Walston J, et al.; Cardiovascular Health Study Collaborative Research Group. Frailty in older adults: evidence for a phenotype. J Gerontol A Biol Sci Med Sci 2001;56:M146-56. https://doi.org/10.1093/ gerona/56.3.m146 


\section{SUPPLEMENTARY MATERIAL}

\section{Members of the GeroCovid Observational Working GRoup ${ }^{16}$ (IN ALPHABETICAL ORDER)}

Angela Marie Abbatecola [ASL Frosinone; RSA INI Città Bianca, Veroli (FR)], Domenico Andrieri [RSA Villa Santo Stefano, S. Stefano di Rogliano (CS)], Sara Antenucci [Ambulatorio Psicogeriatrico, Ortona $(\mathrm{CH})$ ], Rachele Antognoli [Azienda Ospedaliero Universitaria Pisana; RSA Villa Isabella, Pisa], Raffaele Antonelli Incalzi [Università Campus Bio-Medico, Roma], Maria Paola Antonietti [Ospedale Regionale di Aosta], Viviana Bagalà [Azienda Ospedaliero-Universitaria di Ferrara], Giulia Bandini [USL Toscana Centro, Ospedale San Jacopo, Pistoia], Salvatore Bazzano [ULSS 3 Serenissima, Presidio di Dolo (VE)], Giuseppe Bellelli [Ospedale San Gerardo, Monza], Andrea Bellio [Azienda Ospedale Università di Padova], Federico Bellotti [Azienda Ospedaliero-Universitaria di Ferrara], Enrico Benvenuti [USL Toscana Centro, Ospedale Santa Maria Annunziata, Bagno a Ripoli (FI)], Marina Bergamin [Azienda Ospedaliero-Universitaria di Parma], Marco Bertolotti [Azienda Ospedaliero-Universitaria di Modena], Carlo Adriano Biagini [USL Toscana Centro, Ospedale San Jacopo, Pistoia], Angelo Bianchetti [Istituto Clinico Sant'Anna, Brescia], Alessandra Bianchi [Spedali Civili, Montichiari (BS)], Mariangela Bianchi [Policlinico Sant'Orsola-Malpighi, Bologna], Paola Bianchi [Associazione Nazionale Strutture Territoriali e per la Terza Età, Roma], Francesca Biasin [Azienda Ospedale Università di Padova], Silvia Bignamini [Casa di Cura San Francesco, Bergamo], Damiano Blandini [Policlinico Sant'Orsola-Malpighi, Bologna], Stefano Boffelli [Fondazione Poliambulanza, Brescia], Cristiano Bontempi [Azienda Ospedale Università di Padova], Alessandra Bordignon [Azienda Ospedale Università di Padova], Luigi Maria Bracchitta [ATS Milano], Maura Bugada [Casa di Cura San Francesco, Bergamo], Carmine Cafariello [RSA Villa Sacra Famiglia, IHG, Roma; I RSA Geriatria, IHG, Guidonia (RM); III RSA Geriatria, IHG, Guidonia (RM); RSA Estensiva, IHG, Guidonia (RM); RSA Intensiva, IHG, Guidonia $(\mathrm{RM})$ ], Veronica Caleri [USL Toscana Centro, Ospedale San Jacopo, Pistoia], Valeria Calsolaro [Azienda Ospedaliero Universitaria Pisana; RSA Villa Isabella, Pisa], Donatella Calvani [USL Toscana Centro, Presidio Misericordia e Dolce, Prato; USL Toscana Centro, Ospedale Santo Stefano, Prato], Francesco Antonio Campagna [Centro di Riabilitazione San Domenico, Lamezia Terme (CZ)], Andrea Capasso [ASL Napoli 2 Nord], Sebastiano Capurso [RSA Bellosguardo, Civitavecchia $(\mathrm{RM})$ ], Silvia Carino [RSA San Domenico, Lamezia Terme (CZ); Centro di Riabilitazione San Domenico,
Lamezia Terme (CZ); RSA Villa Elisabetta, Cortale (CZ); Casa Protetta Madonna del Rosario, Lamezia Terme (CZ)], Elisiana Carpagnano [Ospedale Giovanni XXIII Policlinico di Bari], Barbara Carrieri [IRCCS INRCA, Ancona], Viviana Castaldo [Presidio Ospedaliero Universitario Santa Maria della Misericordia, Udine], Manuela Castelli [Istituto Geriatrico Camillo Golgi, Abbiategrasso (MI)], Manuela Castellino [Fatebenefratelli, Presidio Ospedaliero Riabilitativo "Beata Vergine Consolata", San Maurizio Canavese (TO)], Alessandro Cavarape [Presidio Ospedaliero Universitario Santa Maria della Misericordia, Udine], Ilaria Cazzulani [Ospedale San Gerardo, Monza], Carilia Celesti [Policlinico Universitario Campus Bio-Medico, Roma], Chiara Ceolin [Azienda Ospedale Università di Padova], Maria Giorgia Ceresini [Azienda Ospedaliero-Universitaria di Ferrara], Arcangelo Ceretti [Istituto Geriatrico Camillo Golgi, Abbiategrasso (MI)], Antonio Cherubini [IRCCS INRCA, Ancona], Anita Chizzoli [Istituto Clinico Sant'Anna, Brescia], Erika Ciarrocchi [IRCCS INRCA, Ancona], Paola Cicciomessere [Azienda Ospedaliero Universitaria di Foggia], Alessandra Coin [Azienda Ospedale Università di Padova], Mauro Colombo [Istituto Geriatrico Camillo Golgi, Abbiategrasso (MI)], Annalisa Corsi [USL Toscana Centro, Ospedale San Jacopo, Pistoia], Antonella Crispino [RSA Villa Santo Stefano, S. Stefano di Rogliano (CS); RSA Villa Silvia, Altilia Grimaldi (CS)], Roberta Cucunato [RSA Villa Santo Stefano, S. Stefano di Rogliano (CS); RSA Villa Silvia, Altilia Grimaldi (CS)], Carlo Custodero [Ospedale Giovanni XXIII Policlinico di Bari], Federica D’Agostino [Casa Sollievo della Sofferenza, S. Giovanni Rotondo (FG)], Maria Maddalena D'Errico [Casa Sollievo della Sofferenza, S. Giovanni Rotondo (FG)], Ferdinando D'Amico [RSA San Giovanni di Dio, Patti (ME); RSA Sant'Angelo di Brolo (ME)], Aurelio De lorio [Azienda Ospedaliero-Universitaria di Parma], Alessandro De Marchi [Policlinico Sant'Orsola-Malpighi, Bologna], Annalaura Dell'Armi [III RSA Geriatria, IHG, Guidonia (RM)], Marta Delmonte [Azienda Ospedaliero-Universitaria di Ferrara], Giovambattista Desideri [Ospedale di Avezzano (AQ)], Miriam Devita [Azienda Ospedale Università di Padova], Evelyn Di Matteo [Policlinico Universitario Campus Bio-Medico, Roma], Emma Espinosa [Azienda Ospedali Riuniti Marche Nord, Fano (PU)], Luigi Esposito [Casa Sollievo della Sofferenza, S. Giovanni Rotondo (FG)], Chiara Fazio [Azienda Ospedaliero-Universitaria di Parma], Christian Ferro [RSA Sant'Angelo di Brolo (ME)], Chiara Filippini [Spedali Civili, Montichiari (BS)], Filippo Fini [Azienda Ospedale Università di Padova], Lucia Fiore [Casa Sollievo della Sofferenza, S. Giovanni Rotondo (FG)], Serafina Fiorillo [ASP Vibo Valentia; RSA 
Madonna delle Grazie, Filadelfia (M); Casa di Riposo Mons. Francesco Luzzi, Acquaro (W); Casa di Riposo Villa Betania, Mileto (V); Casa di Riposo Pietro Rosano, Dasà (V); Casa di Riposo Serena Diocesi, Mileto (V); Alloggio per Anziani Villa Amedeo, Francavilla Angitola (W); Casa Allbergo Villa Fabiola, Monterosso Calabro (W); Casa di Riposo Villa Sara, San Nicola da Crissa (W); Casa di Riposo Don Mottola, Tropea (W); Casa di Riposo San Francesco, Soriano Calabro (W); RSA Anziani, Soriano Calabro (V); Casa di Riposo Suore Missionarie del Catechismo, Pizzo (VV)], Caterina Fontana [Azienda Ospedaliero-Universitaria di Modena], Lina Forte [Ospedale di Avezzano (AQ)], Riccardo Franci Montorzi [Azienda Ospedaliero Universitaria Careggi, Firenze], Carlo Fumagalli [Azienda Ospedaliero Universitaria Careggi, Firenze], Stefano Fumagalli [Azienda Ospedaliero Universitaria Careggi, Firenze], Pietro Gareri [ASP Catanzaro], Pier Paolo Gasbarri [Associazione Nazionale Strutture Territoriali e per la Terza Età, Roma], Antonella Giordano [Azienda Ospedaliero Universitaria Careggi, Firenze], Evelina Giuliani [USL Toscana Centro, Ospedale Santa Maria Annunziata, Bagno a Ripoli (FI)], Roberta Granata [RSA Villa Sacra Famiglia, $I H G$, Roma], Antonio Greco [Casa Sollievo della Sofferenza, S. Giovanni Rotondo (FG)], Nadia Grillo [RSA San Domenico, Lamezia Terme (CZ); Casa di Riposo San Domenico, Lamezia Terme (CZ); RSA Villa Elisabetta, Cortale (CZ)], Antonio Guaita [Istituto Geriatrico Camillo Golgi, Abbiategrasso (MI)], Liana Gucciardino [ASP Agrigento], Andrea Herbst [Azienda Ospedaliero Universitaria Careggi, Firenze], Marilena larrera [RSA Sant'Angelo di Brolo (ME)], Giuseppe lelo [Azienda Ospedaliero-Universitaria di Parma], Valerio Alex Ippolito [Casa Protetta Villa Azzurra, Roseto Capo Spulico (CS)], Antonella La Marca [Casa Sollievo della Sofferenza, S. Giovanni Rotondo (FG)], Umberto La Porta [Azienda Ospedaliero-Universitaria di Parma], Ilaria Lazzari [Policlinico Sant'Orsola-Malpighi, Bologna], Diana Lelli [Policlinico Universitario Campus Bio-Medico, Roma], Yari Longobucco [Azienda Ospedaliero-Universitaria di Parma], Francesca Lubian [Ospedale di Bolzano], Giulia Lucarelli [Azienda Ospedaliero Universitaria Careggi, Firenze; USL Toscana Centro, Ospedale San Jacopo, Pistoia], Flaminia Lucchini [Azienda Ospedaliero Universitaria Careggi, Firenze], Daniela Lucente [Spedali Civili, Montichiari (BS)], Lorenzo Maestri [Policlinico Sant'Orsola-Malpighi, Bologna], Marcello Maggio [Azienda Ospedaliero-Universitaria di Parma], Paola Mainquà [Azienda Ospedali Riuniti Marche Nord, Fano (PU)], Mariangela Maiotti [Ospedale San Giovanni Battista, Foligno (PG)], Alba Malara [RSA San Domenico, Lamezia Terme (CZ); Casa di Riposo Villa Marinella, Amantea (CS); Casa Protetta Madonna del Rosario, Lamezia Terme (CZ); Casa Protetta Villa Azzurra,
Roseto Capo Spulico (CS); Centro di Riabilitazione San Domenico, Lamezia Terme (CZ); RSA Casa Amica, Fossato Serralta (CZ); RSA La Quiete, Castiglione Cosentino (CS); Casa di Riposo San Domenico, Lamezia Terme (CZ); RSA Villa Elisabetta, Cortale (CZ); RSA Villa Santo Stefano, S. Stefano di Rogliano (CS); RSA Villa Silvia, Altilia Grimaldi (CS)], Carlotta Mancini [Azienda Ospedaliero Universitaria Careggi, Firenze], Irene Mancuso [RSA San Giovanni di Dio, Patti (ME)], Eleonora Marelli [Istituto Geriatrico Camillo Golgi, Abbiategrasso (MI)], Alessandra Marengoni [Spedali Civili, Montichiari (BS)], Eleonora Marescalco [Azienda Ospedale Università di Padova], Benedetta Martin [Ospedale di Avezzano (AQ)], Valentina Massa [Casa Sollievo della Sofferenza, S. Giovanni Rotondo (FG)], Giulia Matteucci [Azienda Ospedaliero-Universitaria di Ferrara], Irene Mattioli [Azienda Ospedaliero-Universitaria di Ferrara], Liliana Mazza [Policlinico Sant'Orsola-Malpighi, Bologna], Carmela Mazzoccoli [Ospedale Giovanni XXIII Policlinico di Bari], Fiammetta Monacelli [IRCCS Policlinico San Martino, Genova], Paolo Moneti [RSA Villa Gisella, Firenze], Fabio Monzani [Azienda Ospedaliero Universitaria Pisana; RSA Villa Isabella, Pisa], Federica Morellini [Azienda Ospedaliero-Universitaria di Modena], Maria Teresa Mormile [ASL Napoli 2 Nord], Enrico Mossello [Azienda Ospedaliero Universitaria Careggi, Firenze], Chiara Mussi [Azienda Ospedaliero-Universitaria di Modena], Francesca Maria Nigro [USL Toscana Centro, Ospedale Santo Stefano, Prato], Marianna Noale [RSA AltaVita, Istituzioni Riunite di Assistenza, Padova], Chukwuma Okoye [Azienda Ospedaliero Universitaria Pisana], Giuseppe Orio [Policlinico Sant'Orsola-Malpighi, Bologna], Sara Osso [RSA La Quiete, Castiglione Cosentino (CS)], Chiara Padovan [Azienda Ospedale Università di Padova], Annalisa Paglia [Azienda Ospedaliero Universitaria di Foggia], Giulia Pelagalli [Azienda Ospedaliero Universitaria Careggi, Firenze], Laura Pelizzoni [Policlinico Sant'Orsola-Malpighi, Bologna], Agostino Perri [RSA La Quiete, Castiglione Cosentino (CS)], Maria Perticone [Casa di Riposo Villa Marinella, Amantea (CS)], Giacomo Piccardo [IRCCS Policlinico San Martino, Genova], Alessandro Picci [Presidio Ospedaliero Universitario Santa Maria della Misericordia, Udine], Margherita Pippi [Ospedale San Giovanni Battista, Foligno (PG)], Giuseppe Provenzano [ASP Agrigento], Matteo Pruzzo [IRCCS Policlinico San Martino, Genova], Francesco Raffaele Addamo [RSA San Giovanni di Dio, Patti (ME)], Cecilia Raffaelli [Azienda Ospedale Università di Padova], Francesca Remelli [Azienda Ospedaliero-Universitaria di Ferrara], Onofrio Resta [Ospedale Giovanni XXIII Policlinico di Bari], Antonella Riccardi [Policlinico Sant'Orsola-Malpighi, Bologna], Daniela Rinaldi [Ospedale di Comunità (Camposampiero), Distretto Alta Padovana, ULSS 6 Euganea, 
Padova], Renzo Rozzini [Fondazione Poliambulanza, Brescia], Matteo Rubino [IRCCS Policlinico San Martino, Genova], Carlo Sabbà [Ospedale Giovanni XXIII Policlinico di Bari], Leonardo Sacco [Casa Sollievo della Sofferenza, S. Giovanni Rotondo (FG)], Mariateresa Santoliquido [Casa Sollievo della Sofferenza, S. Giovanni Rotondo (FG)], Mariella Savino [Casa Sollievo della Sofferenza, S. Giovanni Rotondo (FG)], Francesco Scarso [Azienda Ospedaliero-Universitaria Sant'Andrea, Roma], Giuseppe Sergi [Azienda Ospedale Università di Padova], Gaetano Serviddio [Azienda Ospedaliero Universitaria di Foggia], Claudia Sgarito [ASP Agrigento], Giovanni Sgrò [RSA Istituto Santa Maria del Soccorso, Serrastretta (CZ); RSA San Vito Hospital, San Vito sullo Jonio (CZ); Casa Protetta Villa Mariolina, Montauro (CZ); Casa Protetta Villa Sant'Elia, Marcellinara (CZ)], Chiara Sidoli [Ospedale San Gerardo, Monza], Federica Sirianni [Casa di Riposo Villa Marinella, Amantea (CS)], Vincenzo Solfrizzi [Ospedale Giovanni XXIII Policlinico di Bari], Benedetta Soli [Azienda Ospedaliero-Universitaria di Modena], Debora Spaccaferro [RSA Estensiva, IHG, Guidonia (RM); RSA Intensiva, IHG, Guidonia (RM)], Fausto Spadea [RSA Casa Amica, Fossato Serralta (CZ)], Laura Spadoni [Ospedale San Giovanni Battista, Foligno (PG)], Laura Tafaro [Azienda Ospedaliero-Universitaria Sant'Andrea, Roma], Luca Tagliafico [IRCCS Policlinico San Martino, Genova], Andrea Tedde [Azienda Ospedaliero-Universitaria di Modena], Camilla Terziotti [Azienda Ospedale Università di Padova], Giuseppe Dario Testa [USL Toscana Centro, Ospedale San Jacopo, Pistoia], Maria Giulia Tinti [Casa Sollievo della Sofferenza, S. Giovanni Rotondo (FG)], Francesco Tonarelli [USL Toscana Centro, Presidio Misericordia e Dolce, Prato], Elisabetta Tonon [USL Toscana Centro, Ospedale San Jacopo, Pistoia], Caterina Trevisan [Ospedale di Comunità (Camposampiero), Distretto Alta Padovana, ULSS 6 Euganea, Padova; Azienda Ospedale Università di Padova], Rita Ursino [I RSA Geriatria, IHG, Guidonia (RM)], Filomena Vella [Azienda Sanitaria Universitaria Giuliano Isontina, Trieste], Maria Villanova [Azienda Ospedale Università di Padova], Aurora Vitali [Azienda Ospedaliero-Universitaria di Ferrara], Stefano Volpato [Azienda Ospedaliero-Universitaria di Ferrara], Francesca Zoccarato [Azienda Ospedale Università di Padova], Sonia Zotti [Policlinico Universitario Campus Bio-Medico, Roma], Amedeo Zurlo [Azienda Ospedaliero-Universitaria di Ferrara].

\section{Members of the GeroVAX Working Group ${ }^{17}$ (IN ALPHABETICAL ORDER)}

Angela Marie Abbatecola [ASL Frosinone; in collaborazione con RSA INI Città Bianca, Veroli (FR); RSA San Germano, Piedimonte San Germano (FR); RSA Santa
Maria, Castrocielo (FR); RSA Villa degli Ulivi, Sant'Elia Fiumerapido (FR); RSA Villa Letizia, Patrica (FR)], Domenico Andrieri [RSA Villa Santo Stefano, S. Stefano di Rogliano (CS); RSA Villa Silvia, Altilia Grimaldi (CS)], Raffaele Antonelli Incalzi [Università Campus Bio-Medico, Roma], Francesca Arenare [ASP Golgi Redaelli, Istituto Piero Redaelli, Milano], Tatjana Baldovin [Università di Padova], Viviana Bagalà [CRA Capatti, Riva del Po (FE); CRA Mantovani, Copparo (FE); CRA Plattis, Cento (FE); CRA Quisisana, Ostellato (FE); CRA Ripagrande, ASP Ferrara], Riccardo Bernardi [RSA Estensiva per DCCG, IHG, Guidonia (RM)], Alessandra Bianchi [RSA Arici Sega, Fondazione Brescia Solidale, Brescia], Paola Bianchi [Associazione Nazionale Strutture Territoriali e per la Terza Età, Roma], Raffaella Bisceglia [RSA Universo Salute Opera Don Uva, Foggia], Fabio Bontempi [ASP Golgi Redaelli, Istituto Piero Redaelli, Milano], Gilda Borselli [Società Italiana di Gerontologia e Geriatria, Firenze], Luigi Bottaro [ASP Golgi Redaelli, Istituto Piero Redaelli, Milano], Elisa Bottoni [Centro Benessere (Riabilitazione), Frosinone], Silvia Brandi [Casa di Riposo Torriglia, Chiavari (GE)], Claudio Bravin [RSA Fondazione Casa Industria, Brescia], Maria Adele Buizza [Azienda Speciale Casa di Riposo M. Bonincontri, Brescia], Carmine Cafariello [RSA Disabili Alto Mantenimento, IHG, Guidonia (RM); RSA Estensiva per DCCG, $I H G$, Guidonia (RM); RSA Estensiva, IHG, Guidonia (RM); RSA Geriatria Alto Mantenimento, IHG, Guidonia (RM); RSA Geriatria Alto Mantenimento, IHG, Guidonia (RM); RSA Intensivo, IHG, Guidonia (RM); RSA Villa Sacra Famiglia, IHG, Roma], Alessia Maria Calabrese [RSA Bastia, Livorno; RSA Casa Mimosa, Pisa; RSA Coteto, Livorno; RSA Madonna della Fiducia, Calambrone (PI); RSA Pio Istituto Campana, Seravezza (LU); RSA Villa Isabella, Pisa], Valeria Calsolaro [RSA Bastia, Livorno; RSA Casa Mimosa, Pisa; RSA Coteto, Livorno; RSA Madonna della Fiducia, Calambrone (PI); RSA Pio Istituto Campana, Seravezza (LU); RSA Villa Isabella, Pisa], Marta Canepa [RSA Villa Sorriso, Rapallo (GE)], Carla Capasso [RSA Fondazione Casa Industria, Brescia], Mariagrazia Capuano [RSA Gianà, Qualiano (NA)], Sebastiano Capurso [RSA Bellosguardo, Civitavecchia (RM)], Gabriele Carbone [RSA Estensiva per DCCG, IHG, Guidonia (RM)], Marialudovica Carducci [RSA Villa Sacra Famiglia, IHG, Roma], Silvia Carino [Casa di Riposo San Domenico, Lamezia Terme (CZ); Casa Protetta Madonna del Rosario, Lamezia Terme (CZ); Centro di Riabilitazione San Domenico, Lamezia Terme (CZ); RSA San Domenico, Lamezia Terme (CZ); RSA Villa Elisabetta, Cortale (CZ)], Nicoletta Cattaneo [RSA Sandro Pertini, ASST Rhodense, Garbagnate Milanese (MI)], Francesco Ceravolo [Casa Protetta San Domenico, Palermiti (CZ); RSA Santa Maria del Monte, Petrizzi (CZ)], Maria Angelica Dorotea Chiesara [RSA 
San Pietro, Monza], Danila Clerici [RSA Sandro Pertini, ASST Rhodense, Garbagnate Milanese (MI)], Pierpaolo Clerici [ASST Ovest Milanese, Legnano (MI)], Alessandra Coin [Azienda Ospedale Università Padova], Vieri Collacchioni [RSA Nuova Villa Rio, San Godenzo (FI)], Mauro Colombo [ASP Golgi Redaelli, Istituto Piero Redaelli, Milano], Michela Compiano [RSA Le due Palme, Sestri Levante (GE)], Giuseppina Costanza [RSA Villa delle Palme, Villafrati (PA)], Giovanna Crupi [RSA2 Sindromi da immobilizzazione, ASP Palermo], Roberta Cucunato [RSA Villa Santo Stefano, S. Stefano di Rogliano (CS); RSA Villa Silvia, Altilia Grimaldi (CS)], Manuela Marina D'Abramo [RSA L'Ulivo, Fondazione Cittadella della Carità, Taranto], Emilia D'Agostino [Casa Protetta San Giuseppe, San Sosti (CS)], Ferdinando D'Amico [RSA San Giovanni di Dio, Patti (ME); RSA Sant'Angelo di Brolo (ME)], Stefania Del Vecchio [RSA Bastia, Livorno; RSA Casa Mimosa, Pisa; RSA Coteto, Livorno; RSA Madonna della Fiducia, Calambrone (PI); RSA Pio Istituto Campana, Seravezza (LU); RSA Villa Isabella, Pisa], Maria Deleo [RSA Villa delle Palme, Villafrati (PA)], Annalaura Dell'Armi [RSA Geriatria Alto Mantenimento, IHG, Guidonia (RM)], Tommasina Di Brango [RSA Santa Maria, Castrocielo (FR)], Anna Di Lonardo [Istituto Superiore di Sanità, Roma], Maria Raffaella Di Nanno [RSA II Sorriso, Sanità Più, Foggia; RSA Universo Salute Opera Don Uva, Foggia], Babette Dijk [RSA Estensiva, ASL4, Chiavari (GE)], Luisa Elmo [RSA Villa Sorriso, Marano sul Panaro (MO)], Giorgio Fedele [Istituto Superiore di Sanità, Roma], Marisa Ferraro [RSA II Sorriso, Sanità Più, Foggia], Christian Ferro [RSA Sant'Angelo di Brolo (ME)], Claudia Fiorucci [RSA Estensiva, IHG, Guidonia (RM); RSA Intensivo, IHG, Guidonia (RM)], Francesca Fortunato [Università di Foggia], Pasquale Froncillo [RSA Dimora Marigold, Pozzuoli (NA)], Domenico Galasso [Casa Protetta San Domenico, Palermiti (CZ); RSA Santa Maria del Monte, Petrizzi (CZ)], Nicola Galdiero [RSA Gianà, Qualiano (NA)], Caterina Galdiero [RSA Gianà, Qualiano (NA)], Stefania Gallo [RSA Sandro Pertini, ASST Rhodense, Garbagnate Milanese (MI)], Pier Paolo Gasbarri [Associazione Nazionale Strutture Territoriali e per la Terza Età, Roma], Maria Grazia Gennai [RSA Nuova Villa Rio, San Godenzo (Fl)], Giuliana Ghiselli Ricci [RSA Villa Sorriso, Marano sul Panaro (MO)], Elisa Giribaldi [Casa di Riposo Torriglia, Chiavari (GE)], Carmen Godeanu [CRA Villa dei Ciliegi, Valsamoggia (BO)], Roberta Granata [RSA Villa Sacra Famiglia, IHG, Roma], Giada Ida Greco [RSA La Quiete, Castiglione Cosetino (CS)], Angela Greco [RSA2 Sindromi da immobilizzazione, ASP Palermo], Antonio Grillo [ASP Golgi Redaelli, Istituto Piero Redaelli, Milano], Gianbattista Guerrini [RSA Arici Sega, Fondazione Brescia Solidale, Brescia], Mauro Guglielmo [RSA Sandro Pertini, ASST Rhodense, Garbagnate Milanese
(MI)], Labjona Haxhiaj [RSA AltaVita, Istituzioni Riunite di Assistenza, Padova], Claudio Giuseppe lacovella [RSA Santa Maria, Castrocielo (FR)], Marina Indino [RSA Villaggio Amico, Gerenzano (VA)], Valerio Alex Ippolito [Casa Protetta Villa Azzurra, Roseto Capo Spulico (CS)], David Kanah [ASP Golgi Redaelli, Istituto Piero Redaelli, Milano], Liudmila Kountsevich [RSA Disabili Alto Mantenimento, IHG, Guidonia (RM)], Jovan Leci [CRA Villa Teresa, Sasso Marconi (BO)], Agata Lipari [Casa di Riposo Domus Aurea, Africo (RC); Casa Protetta Universo, Africo (RC); RSA Universo, Africo (RC)], Vincenzo Longo [Casa di Riposo Domus Aurea, Africo (RC); Casa Protetta Universo, Africo (RC); RSA Universo, Africo $(R C)$ ], Stefania Maggi [Consiglio Nazionale delle Ricerche, Padova], Alba Malara [Casa di Riposo San Domenico, Lamezia Terme (CZ); Casa di Riposo Villa Marinella, Amantea (CS); Casa Protetta Madonna del Rosario, Lamezia Terme (CZ); Casa Protetta Villa Azzurra, Roseto Capo Spulico (CS); Centro di Riabilitazione San Domenico, Lamezia Terme (CZ); RSA Casa Amica, Fossato Serralta (CZ); RSA La Quiete, Castiglione Cosetino (CS); RSA San Domenico, Lamezia Terme (CZ); RSA Villa Elisabetta, Cortale (CZ); RSA Villa Santo Stefano, S. Stefano di Rogliano (CS); RSA Villa Silvia, Altilia Grimaldi (CS)], Leonarda Maltese [RSA Bastia, Livorno; RSA Casa Mimosa, Pisa; RSA Coteto, Livorno; RSA Madonna della Fiducia, Calambrone (PI); RSA Pio Istituto Campana, Seravezza (LU); RSA Villa Isabella, Pisa], Maria Marotta [RSA Villa Letizia, IHG, Patrica (FR)], Giuseppe Mazzarella [RSA Villa degli Ulivi, Sant'Elia Fiumerapido (FR)], Hior Melnik [Casa di Riposo Domus Aurea, Africo (RC); Casa Protetta Universo, Africo (RC); RSA Universo, Africo (RC)], Pasquale Minchella [Azienda Ospedaliera Pugliese Ciaccio, Catanzaro], Paolo Moneti [RSA Villa Gisella, Firenze], Fabio Monzani [RSA Bastia, Livorno; RSA Casa Mimosa, Pisa; RSA Coteto, Livorno; RSA Madonna della Fiducia, Calambrone (PI); RSA Pio Istituto Campana, Seravezza (LU); RSA Villa Isabella, Pisa], Walter Morandotti [ASP Golgi Redaelli, Istituto Piero Redaelli, Milano], Francesco Morelli [RSA Villa San Giuseppe, Crotone], Maria Grazia Mortola [RSA Villa Sorriso, Rapallo (GE)], Marianna Noale [RSA Opera Immacolata Concezione, Padova], Chukwuma Okoye [RSA Bastia, Livorno; RSA Casa Mimosa, Pisa; RSA Coteto, Livorno; RSA Madonna della Fiducia, Calambrone (PI); RSA Pio Istituto Campana, Seravezza (LU); RSA Villa Isabella, Pisa], Graziano Onder [Istituto Superiore di Sanità, Roma], Patrizia Orlanducci [CRA Villa dei Ciliegi, Valsamoggia (BO); CRA Villa Teresa, Sasso Marconi (BO)], Barbara Paganelli [RSA Bosco in Città, Brugherio (MB); RSA Scaccabarozzi, Ornago (MB)], Michele Pagano [RSA Buon Pastore Onlus, Palermo], Nicola Pagano [RSA Quadrifoglio, Giugliano in Campania (NA)], Raffaele Palladino [RSA II Sorriso, 
Sanità Più, Foggia; RSA Universo Salute Opera Don Uva, Foggia], Annapina Palmieri [Istituto Superiore di Sanità, Roma], Magda Palumeri [RSA Buon Pastore Onlus, Palermo], Simone Paolini [RSA AltaVita, Istituzioni Riunite di Assistenza, Padova; RSA Opera Immacolata Concezione, Padova], Raimondo Paternò [Casa di Riposo Domus Aurea, Africo (RC); Casa Protetta Universo, Africo (RC); RSA Universo, Africo (RC)], Angela Pavan [ASP Golgi Redaelli, Istituto Piero Redaelli, Milano], Loris Pelucchi [RSA Sandro Pertini, ASST Rhodense, Garbagnate Milanese (MI)], Agostino Perri [RSA La Quiete, Castiglione Cosetino (CS)], Francesco Perticone [Casa Protetta San Domenico, Palermiti (CZ); RSA Santa Maria del Monte, Petrizzi (CZ)], Rosanna Pesce [RSA Quadrifoglio, Giugliano in Campania (NA)], Sabrina Pigozzo [CRA di Cittadella (PD)], Francesco Pili [Casa Protetta San Teodoro, Crotone; Casa Protetta Villa del Rosario, Crotone; RSA San Teodoro, Crotone; RSA Villa del Rosario, Crotone], Rosa Prato [Università di Foggia], Rosanna Pullia [RSA Bastia, Livorno; RSA Casa Mimosa, Pisa; RSA Coteto, Livorno; RSA Madonna della Fiducia, Calambrone (PI); RSA Pio Istituto Campana, Seravezza (LU); RSA Villa Isabella, Pisa], Ahmad Amedeo Qasem [RSA Bastia, Livorno; RSA Casa Mimosa, Pisa; RSA Coteto, Livorno; RSA Madonna della Fiducia, Calambrone (PI); RSA Pio Istituto Campana, Seravezza (LU); RSA Villa Isabella, Pisa], Francesco Raffaele Addamo [RSA San Giovanni di Dio, Patti (ME)], Cecilia Raffaelli [RSA AltaVita, Istituzioni Riunite di Assistenza, Padova], Vincenzo Restivo [Università di Palermo], Michela Fernanda Rigon [RSA Opera Immacolata Concezione, Padova], Franco Romagnoni [CRA Capatti, Riva del Po (FE); CRA Mantovani, Copparo (FE); CRA Plattis, Cento (FE); CRA Quisisana, Ostellato (FE); CRA Ripagrande, ASP Ferrara], Carmine Romaniello [RSA INI Città Bianca, Veroli (FR)], Valentina Romano [RSA Fondazione Casa Industria, Brescia], Maria Cristina Ruberto [Casa Protetta San Giuseppe,
San Sosti (CS)], Marcello Russo [ASL Frosinone], Bruno Sala [RSA Luigi Accorsi, Legnano (MI)], Sara Sambo [CRA di Cittadella (PD); RSA AltaVita, Istituzioni Riunite di Assistenza, Padova], Maria Concetta Sciurti [ASP Golgi Redaelli, Istituto Piero Redaelli, Milano], Antonietta Scriva [Casa di Riposo Domus Aurea, Africo (RC); Casa Protetta Universo, Africo (RC); RSA Universo, Africo $(\mathrm{RC})$ ]; Luca Secchi [RSA Villa Sorriso, Marano sul Panaro (MO)], Vincenzo Settembrini [Casa Protetta Villa Azzurra, Roseto Capo Spulico (CS)], Federica Sirianni [Casa di Riposo Villa Marinella, Amantea (CS); RSA Villa Santo Stefano, S. Stefano di Rogliano (CS); RSA Villa Silvia, Altilia Grimaldi (CS)], Deborah Spaccaferro [RSA Estensiva, $I H G$, Guidonia (RM); RSA Intensivo, $I H G$, Guidonia (RM)], Fausto Spadea [RSA Casa Amica, Fossato Serralta (CZ)], Manuela Stefanelli [RSA Villa Sacra Famiglia, IHG, Roma], Paola Stefanelli [Istituto Superiore di Sanità, Roma], Brunella Stelitano [Casa di Riposo Domus Aurea, Africo (RC); Casa Protetta Universo, Africo $(\mathrm{RC})$; RSA Universo, Africo $(\mathrm{RC})]$ ], Stefania Stringhini [RSA Villaggio Amico, Gerenzano (VA)], Andrea Tarsitano [RSA Medicalizzata San Raffaele, Castiglione Cosentino (CS); RSA San Raffaele, Castiglione Cosentino (CS)], Caterina Trevisan [CRA di Cittadella (PD); RSA AltaVita, Istituzioni Riunite di Assistenza, Padova; RSA Opera Immacolata Concezione, Padova], Rita Ursino [RSA Geriatria Alto Mantenimento, IHG, Guidonia (RM)], Giovanni Veneziano [ASP di Palermo], Maria Teresa Vigliotta [RSA San Germano, Piedimonte San Germano (FR)], Marco Vignati [RSA Sandro Pertini, ASST Rhodense, Garbagnate Milanese (MI)], Eva Vignola [RSA Villa Gisella, Firenze], Enrico Virgilio [CRA di Cittadella (PD)], Maria Visconti [RSA Gianà, Qualiano (NA)], Stefano Volpato [Università di Ferrara], Susanna Vozzi [RSA Villaggio Amico, Gerenzano (VA)], Sabrina Zaccone [Casa Protetta San Domenico, Palermiti (CZ); RSA Santa Maria del Monte, Petrizzi (CZ)]. 